\title{
Genomic analysis of Cryaa-R49C and Cryab-R120G knockin mutant mice: 10-year follow-up
}

\author{
Fred Kolling IV ${ }^{1}$, Carol Ringelberg ${ }^{1}$, Mia Wallace ${ }^{2}$, and Usha Andley ${ }^{3 *}$
}

${ }^{1}$ DartMouse, Mouse Speed Congenic Core Facility, Geisel School of Medicine at

Dartmouth, Lebanon, NH 03756, USA

${ }^{2}$ Departments of Pediatrics Washington University School of Medicine, St. Louis, MO 63110, USA

${ }^{3}$ Department of Ophthalmology and Visual Sciences, Washington University School of Medicine, St. Louis, MO 63110, USA

${ }^{*}$ Correspondence: Usha P. Andley, Ph.D.

Department of Ophthalmology and Visual Sciences

660 South Euclid Avenue, Campus Box 8096

Washington University School of Medicine

St. Louis MO 63110 USA

Telephone: (314)362-7167 andley@wustl.edu 
bioRxiv preprint doi: https://doi.org/10.1101/2020.10.16.343186; this version posted October 16, 2020. The copyright holder for this preprint (which was not certified by peer review) is the author/funder. All rights reserved. No reuse allowed without permission.

Co-author e-mails: Fred Kolling IV: Fred.W.Kolling.IV@dartmouth.edu; Carol

Ringelberg: Carol.S.Ringelberg@dartmouth.edu; Mia Wallace: mia@wustl.edu 
1

2

3

4

5

Keywords: Mouse genome, SNP markers, cryaa, cryab, mutation, knockin mice

\section{Abstract}

Objective: Two experimental samples from a mouse line containing Cryaa or Cryab modifications on a predominantly C57BI/6 background using 129Sv mouse strain embryonic stem cells were investigated. The objective was to reexamine the precise genetic background of the mice 10 years after they were converted to the C57BI/6 background.

Results: The genetic backgrounds of the mice were assessed at the DartMouse ${ }^{\mathrm{TM}}$ Speed Congenic Core Facility at the Geisel School of Medicine at Dartmouth.

DartMouse used the Illumina, Inc. Infinium Genotyping Assay to interrogate a custom panel of 5307 SNPs that were spread throughout the genome. The raw SNP data were analyzed using the DartMouse SNaP-Map ${ }^{\mathrm{TM}}$ and Map-Synth ${ }^{\mathrm{TM}}$ software, which allowed for genetic background identification at each SNP location for every mouse. As part of the analysis, 323 SNPs were eliminated from the data prior to generating chromosome maps due to internal quality control protocols. Of the remaining 4984 SNPs, $44.56 \%$ were uninformative (not polymorphic between the two relevant genetic backgrounds) and approximately $0.91 \%$ gave uninterpretable data. The remaining $54.53 \%$ of the returned SNPs were well distributed throughout the genome. The genetic backgrounds were determined to be $98-99 \%$ C57BI/6J, which was the desired background. 


\section{Introduction}

23 Human cataracts are associated with crystallin gene mutations. Mouse models have

24 been extensively used to investigate cataract etiology, mechanism, and phenotype.

25 Many gene targeting models employ embryonic stem cells that are derived from the

26 129Sv mouse strain, which carries a mutation in the phakinin gene (CP49). CP49 and

27 its interaction partner, filensin, produce the lens fiber cell-specific intermediate filament

28 known as the beaded filament $(1,2)$. Importantly, the mutation in CP49 that is carried by

29 the $129 \mathrm{~Sv}$ strain is associated with beaded filaments $(3,4)$. Experiments with CP49-

30 deleted, knockout mice (5) demonstrated that an absence of beaded filaments leads to

31 reduced lens optical clarity (2). Additionally, the cytoskeletal elements found in the lens

32 including actin, vimentin, and intermediate filaments are known to interact with a-

33 crystallin, which is a molecular chaperone that is abundantly expressed in the lens (6).

34 The aggregation of cytoskeletal proteins such as actin, vimentin, GFAP, and tubulin is

35 inhibited by a-crystallin (6).

36 To study the effects of Cryaa and Cryab mutations on cataract formation, we generated

37 mice using 129Sv mouse embryonic stem cells $(7,8)$. The mice were then initially bred with $\mathrm{C} 57 \mathrm{BI} / 6 \mathrm{~J}$ mice for 3 generations to generate a colony with a mixed C57BI/6J/129Sv

39 background. Subsequently, we used speed congenics to convert the Cryaa and Cryab knockin mice to the $\mathrm{C} 57 \mathrm{BI} / 6 \mathrm{~J}$ background. Analyses that were performed 10 years ago

41 found $>95 \%$ C57 background in these mice. Here, we characterized the same mice

42 after numerous generations of breeding with $\mathrm{C} 57 \mathrm{BI} / 6 \mathrm{~J}$ strain mice. Chromosomal

43 analysis of the genomes from two Cryaa and Cryab heterozygous knockin mice from 
44 our present colony are described. Their genomes were characterized and a high

45 percentage of C57 markers persisted (98-99\%).

\section{Main text}

\section{Methods}

Mice were backcrossed for 5 generations using a marker assisted selection (i.e. "speed congenic") approach. The mouse genomes were assessed at the DartMouse ${ }^{\mathrm{TM}}$ Speed

52 Congenic Core Facility at the Geisel School of Medicine at Dartmouth. DartMouse used

53 the Illumina, Inc. (San Diego, CA) Infinium Genotyping Assay to interrogate a custom

54 panel of 5307 SNPs that were spread throughout the genome. The raw SNP data were 55 analyzed using the DartMouse SNaP-Map ${ }^{\mathrm{TM}}$ and Map-Synth ${ }^{\mathrm{TM}}$ software, which

56 determined the genetic background at each SNP location for every mouse. The genetic 57 background at the final back-cross generation was determined.

58 Starting in 2008, the Cryaa-R49C and Cryab-R120G mouse lines have been

59 propagated at the Mouse Genetics Core in the Department of Pediatrics at Washington

60 University School of Medicine. Mice were either mated with siblings of the same

61 genotype, or with C57BI/6J WT mice. In 2015, the Cryaa-R49C and Cryab-R120G mice were re-derived from frozen sperm because we suspected a viral infection in the mouse

63 colonies at the mouse housing facility (based on PCR assays on sentinel mice at the 64 facility). Mice were euthanized by $\mathrm{CO}_{2}$ inhalation. 
Raw data were used to create a detailed chromosome map for each mouse. The chromosome maps were then used to identify the genetic background of each SNP loci throughout the genome using SNaP-Map software. Map-Synth software was used to graphically display the percentage of the mouse genomes that corresponded to each genetic background. From these graphs and maps, the genetic purity of the speed congenic projects that generated our mouse line was verified (background checks).

All raw SNP genotyping data are presented in an excel file in Supplementary materials (Table S1).

\section{Results}

In the current study, we analyzed two mice. Mouse 1336 had one WT Cryaa allele and one Cryaa-R49C mutant allele. The second mouse (1396) had one WT Cryab allele and one Cryab-R120G mutant allele. We previously reported the effects of these mutations on cataract formation (7-11).

For analysis purposes, we assumed that the genetic background of our experimental samples was a mix of $\mathrm{C} 57 \mathrm{BI} / 6$ and $129 \mathrm{~Sv}$ with no other contaminating genetic backgrounds. In addition, 323 SNPs were eliminated from our data prior to generating chromosome maps due to internal quality control measures. Of the remaining 4984 SNPs, $44.56 \%$ were uninformative (i.e. not polymorphic between the two relevant genetic backgrounds) and approximately $0.91 \%$ yielded uninterpretable data. The remaining $54.53 \%$ of SNPs returned useful data.

Figure 1 displays the chromosome maps of C57BI/6J, 129Sv, Cryaa-R49C-het knockin, and Cryab-R120G-het knockin mice. Our analysis showed that these mice had 99.6\% 
87 and $98.7 \% \mathrm{C} 57 \mathrm{Bl} / 6$ genomes (Figure 2). Interestingly, the segments of the genomes that showed the highest 129Sv background were located on chromosomes where the mutation was created. For example, in the case of the Cryaa-R49C-het mouse 1336, the areas indicated by the purple bands occurred on chromosome 17 , which is the mouse chromosome where the Cryaa gene is located. Similarly, in the Cryab-R120G-

92 het mouse 1396, the purple bands, which indicate the 129Sv and C57BI backgrounds, occurred on chromosome 9, where the Cryab gene is located in the mouse genome.

94 This type of analysis demonstrates the power of the SNaP-Map software for assigning each marker to the proper genetic background. The current analysis duplicates a similar analysis that was performed in 2010 by DartMouse on earlier generations of the same mouse lines. In the 2010 analysis, after backcrossing and speed congenics, the C57BI/6 background was 97\% in Cryaa-R49C het mice and 95.8\% in Cryab-R120G-het mice. The repeated analysis in 2019, after multiple breeding cycles and re-derivation from frozen sperm, indicated that the C57BI/6 background percentage had increased by 23\%. The background was $99.6 \%$ and $98.7 \%$ C57BI/6 for Cryaa-R49C-het and CryabR120G-het mice, respectively (Figure 2A).

103 Further analysis of the same data showed that the Cryaa-R49C-het knockin and Cryab104 R120G-het knockin mice had 99.0\% and 97.01\% C57BI/6J backgrounds (Figure 2B). A 105 comparison of the $\mathrm{C} 57 \mathrm{BI} / 6 \mathrm{~J}$ and $\mathrm{C} 57 \mathrm{BI} / 6 \mathrm{~N}$ chromosome maps is displayed in Figure 3. 106 The full SNP panel contained 201 distinct markers, which distinguished the C57BI/6 "J" 107 from the C57BI/6 "N" sub-strains. We analyzed these SNP markers to determine the J 108 and $\mathrm{N}$ strain percentages in the Cryaa and Cryab knockin mice. Chromosome maps 
109 that include these SNPs were analyzed to distinguish between the B6J and B6N sub-

110 strains (Figure 3).

111 Of the 5307 loci on our SNP panel, 83 fell outside of the DartMouse 95\% quality control

112 confidence interval and were not referenced in the analyses. Additionally, as displayed

113 in Table S2 (Supplementary materials), a small percentage of the SNPs that were

114 interrogated returned uninterpretable data. Historically, approximately $1 \%$ of the total

115 panel of SNPs that are investigated using DartMouse protocols return non-useable

116 data. These were located throughout the genome and are shown in Figures 1 and 3.

118 Discussion

119 The 129Sv mouse strain carries a mutation in the gene for CP49 (phakinin) and also 120 lacks CP49, which is an intermediate filament protein that is specific to lens fiber cells.

121 Consequently, 129Sv mice are almost completely deficient in CP49 protein and its

122 interaction partner, filensin, which results in the absence of the beaded filament in the

123 lens. The $129 \mathrm{~Sv}$ mouse strain exhibits a slow and progressive loss of optical clarity. In

124 addition, the lens cytoskeleton interacts with aB-crystallin, and therefore, the absence of

125 the beaded filament can confound interpretations of aB-crystallin mutation effects. Thus,

126 we sought to convert these mice to the C57BI/6J background, which possesses a

127 normal CP49 gene level (4).

128 Many knockout and knockin mouse models utilize embryonic stem cells from the 129Sv 129 strain. This can confound the interpretation of cataracts that occur as a result of other 
gene mutations and is especially complicated when exploring genes, including crystallin, that are known to interact with lens beaded filaments.

Here, we sought to establish the $\mathrm{C} 57 \mathrm{BI} / 6 \mathrm{~J}$ background in mice that had mutations in the human cataract-linked Cryaa and Cryab genes. These mice were backcrossed with C57BL/6J mice by speed congenics. Lens fiber cells that lack CP49 are more pliable than stiffer fiber cells derived from WT mice. The cells that lack CP49 (CP49 mutant) also have a higher axial to equatorial diameter ratio (3). Notably, 129Sv embryonic stem cells are often used in gene targeting studies even though the presence of a CP49 mutation confounds the cataract phenotype. Although the lens fiber cells in the $129 \mathrm{~Sv}$ strain mice initially appear to differentiate and elongate normally, the 129Sv phenotype develops slowly, and a progressive loss of lens optical clarity is detectable by electron microscopy.

The absence of CP49, the near absence of filensin, and the resultant absence of the beaded filament may have downstream effects on any proteins or structures that are binding partners with these proteins. Thus, the true ramifications of the CP49 mutation in past gene-targeting studies may be difficult to assess. To eliminate the possibility that the CP49 mutation is affecting the results, it may be necessary to backcross the target gene to a wild-type background.

The speed congenics methods that we describe, combined with SNP and chromosome analyses, can be applied to other mouse models. An Rd8 mutation has been found in the $\mathrm{C} 57 \mathrm{BI} / 6 \mathrm{~N}$ mouse strain and produces retinal phenotypes (12). The speed congenics 
152 method used in this study could be useful to convert those mice to a more suitable

153 genetic background. Using genomic analysis of the Cryaa and Cryab knockin mouse

154 chromosomes, we demonstrated that these mice have C57 characteristics instead of

155 129Sv strain traits. The genomes were 97 and 99\% C57BI/6J. Thus, cataracts observed

156 in these knockin mice are not confounded by the presence of the phakinin mutation and

157 are exclusively due to Cryaa or Cryab mutations.

158 The 129Sv mouse strain is widely used as a source of embryonic stem cells for

159 generating knockin and knockout mouse models for cataract lens studies. Starting in

160 2007, we backcrossed knockin mice that had Cryaa or Cryab mutations with C57BI/6J

161 mice for 3 generations. Subsequently, we used speed congenics in 2009-2010 to

162 generate mice that had a higher purity C57BI/6J background. Here, we report the same

163 analysis that was completed in 2010, but are updating the analysis in 2019 to include

164 mice that were sib-mated for many generations over the years. The C57BI/6J

165 background mouse models still produced cataracts, which suggests that the cataracts

166 are unlikely to be caused by the CP49 mutation.

\section{Limitations}

168 We assumed that the genetic background of the experimental samples was a mix of

$169 \mathrm{C} 57 \mathrm{BI} / 6$ and $129 \mathrm{~Sv}$, with no other contaminating backgrounds.

\section{List of Abbreviations}

171 SNP, single-nucleotide polymorphism; Wild-type, WT.

\section{Declarations}




\section{Ethics approval and consent to participate}

174 All animal experiments were approved by the Washington University in St. Louis IACUC

175 Committee (Protocol number 20170212). No human studies were conducted in this

176 research.

177 Consent for publication

178 Each author has agreed to publication of this manuscript.

\section{Availability of data and materials}

180 All data generated or analyzed during this study are included in the published article

181 and supplementary information files.

182 Competing interests

183 The authors declare that they have no competing interests.

184 Funding

185 This work was supported by the National Eye Institute of the National Institutes of

186 Health under award numbers R01 EY05681-33 (to UPA) and Core grant P30

187 EY002687, and an unrestricted grant to the Washington University in St. Louis School

188 of Medicine Department of Ophthalmology and Visual Sciences from Research to

189 Prevent Blindness. DartMouse is supported by NCl Cancer Center Support Grant

190 5P30CA023108 and NIH S10 Instrumentation Grant OD021667.

\section{Authors' contributions}


192 FK: Data acquisition, data analysis, data interpretation, report writing; CR: Data 193 acquisition, data analysis, data interpretation, report writing; MW: Conception and 194 design of the work, manuscript writing; UPA: Manuscript writing, funding procurement.

195 All authors have approved the submitted version of the manuscript and have agreed to 196 be both personally accountable for their own contributions and to ensure that questions 197 related to the accuracy or integrity of any part of the work, even ones in which the 198 author was not personally involved, are appropriately investigated and resolved and that 199 the resolution is documented in the literature.

\section{Acknowledgements}

201 Not applicable. 


\section{REFERENCES}

203 1. Hess JF, Casselman JT, FitzGerald PG. cDNA analysis of the $49 \mathrm{kDa}$ lens fiber

204 cell cytoskeletal protein: a new, lens-specific member of the intermediate filament

205 family? Curr Eye Res. 1993;12(1):77-88.

206 2. Alizadeh A, Clark J, Seeberger T, Hess J, Blankenship T, FitzGerald PG.

207 Characterization of a mutation in the lens-specific CP49 in the 129 strain of mouse.

208 Invest Ophthalmol Vis Sci. 2004;45(3):884-91.

209 3. Fudge DS, McCuaig JV, Van Stralen S, Hess JF, Wang H, Mathias RT, et al.

210 Intermediate filaments regulate tissue size and stiffness in the murine lens. Invest

211 Ophthalmol Vis Sci. 2011;52(6):3860-7.

212 4. Yoon KH, Blankenship T, Shibata B, Fitzgerald PG. Resisting the effects of

213 aging: a function for the fiber cell beaded filament. Invest Ophthalmol Vis Sci.

$2142008 ; 49(3): 1030-6$.

215 5. Alizadeh A, Clark JI, Seeberger T, Hess J, Blankenship T, Spicer A, et al.

216 Targeted genomic deletion of the lens-specific intermediate filament protein CP49.

217 Invest Ophthalmol Vis Sci. 2002;43(12):3722-7.

218 6. Nicholl ID, Quinlan RA. Chaperone activity of alpha-crystallins modulates

219 intermediate filament assembly. Embo J. 1994;13(4):945-53.

2207 Andley UP, Hamilton PD, Ravi N. Mechanism of insolubilization by a single-point

221 mutation in alphaA-crystallin linked with hereditary human cataracts. Biochemistry.

$222 \quad$ 2008;47(36):9697-706. 
223 8. Andley UP, Hamilton PD, Ravi N, Weihl CC. A knock-in mouse model for the

224 R120G mutation of alphaB-crystallin recapitulates human hereditary myopathy and

225 cataracts. PLoS One. 2011;6(3):e17671.

2269 . Andley UP, Reilly MA. In vivo lens deficiency of the R49C alphaA-crystallin

227 mutant. Exp Eye Res. 2010;90(6):699-702.

228 10. Andley UP. AlphaA-crystallin R49Cneo mutation influences the architecture of 229 lens fiber cell membranes and causes posterior and nuclear cataracts in mice. BMC

230 Ophthalmol. 2009;9:4.

231 11. Reilly MA, Andley UP. Quantitative biometric phenotype analysis in mouse

232 lenses. Mol Vis. 2010;16:1041-6.

233 12. Mattapallil MJ, Wawrousek EF, Chan CC, Zhao H, Roychoudhury J, Ferguson

234 TA, et al. The Rd8 mutation of the Crb1 gene is present in vendor lines of $\mathrm{C} 57 \mathrm{BL} / 6 \mathrm{~N}$

235 mice and embryonic stem cells, and confounds ocular induced mutant phenotypes.

236 Invest Ophthalmol Vis Sci. 2012;53(6):2921-7. 
242 Figure 1. Chromosome maps displaying areas of C57BI/6 and $129 \mathrm{~Sv}$ heterozygosity 243 and homozygosity in Cryaa-R49C and Cryab-R120G mice. Chromosome numbers are 244 shown along the top of each map. Chromosome 20 and 21 are the sex chromosomes 245 ( $X$ and $Y$ respectively). Each of the SNP markers in their physical location within the 246 genome of the mouse is shown. These SNP markers are color coded to visually depict 247 the genetic origin as either the "Donor" strain or the "Recipient" strain. Green, C57BI/6;

248 Purple, Heterozygous (C57BI/6 x 129Sv); Orange: 129Sv. The knockin modification can 249 be clearly identified on the proximal ends of chromosomes 17 and 9 for the Cryaa-R49C 250 and Cryab-R120G mice, respectively.

Figure 2. Graphs displaying genetic material percentages. (A) C57BI/6 or $129 \mathrm{~Sv}$ in Cryaa-R49C mouse 1336 and Cryab-R120G mouse 1396. (B) C57BI/6J or C57BI/6N in Cryaa-R49C mouse 1336 and Cryab-R120G mouse 1396. (Part of the graph in (A) was 255 published in Invest Ophthalmol Vis Sci. 2019; 60: 3320-3331. Copyright Association for Research in Vision and Ophthalmology. https://doi.org/10.1167/iovs.18-25647).

Figure 3. Maps displaying areas of C57/BI6J and C57BI/6N heterozygosity and homozygosity in Cryaa-R49C and Cryab-R120G mice. Each of the SNP markers in their physical location within the mouse genome is shown. These SNP markers are color coded to visually depict the genetic origin as either the C57BI/6J or C57BI/6N strain. 
262 Green, C57BI/6J homozygous; Purple, Heterozygous (C57BI/6 x C57BI/6N); Orange:

263 C57BI/6N homozygous.

264 Supplementary Material

265 Table S1: Binary raw data interrogated from the analysis of Cryaa-R49C and Cryab-

266 R120G mice.

267

268 Table S2: Summary of SNP analysis of Cryaa-R49C and Cryab-R120G mice. 
A

\section{$\mathrm{Chr}$}

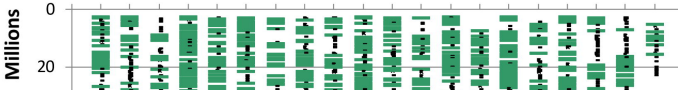

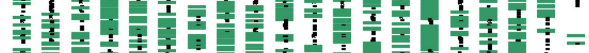

400 i

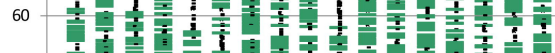
늘 1

80 1

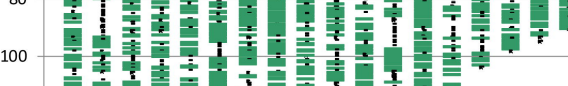

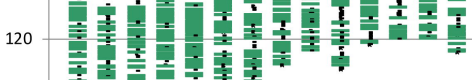

140

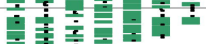

160 $\div \pm$

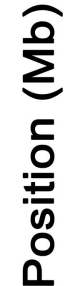

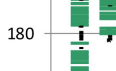

i

C

$\mathrm{C} 57 \mathrm{BI} / 6$

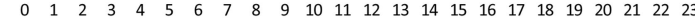

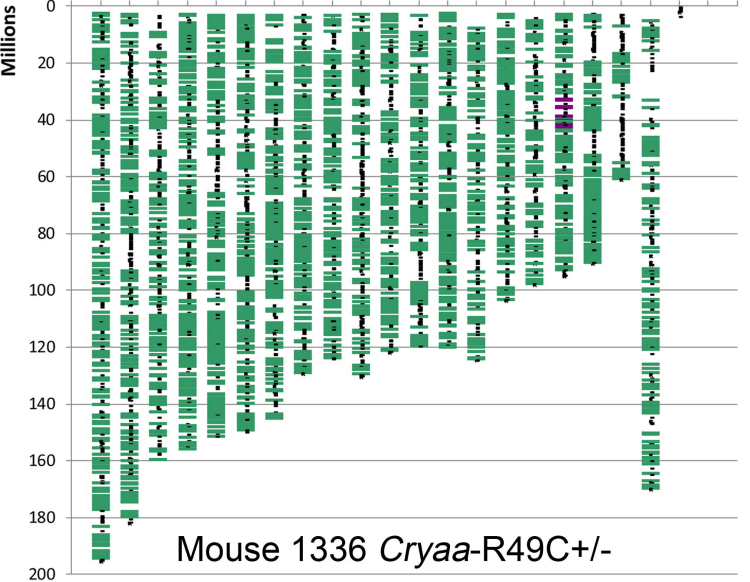

\section{Chr}
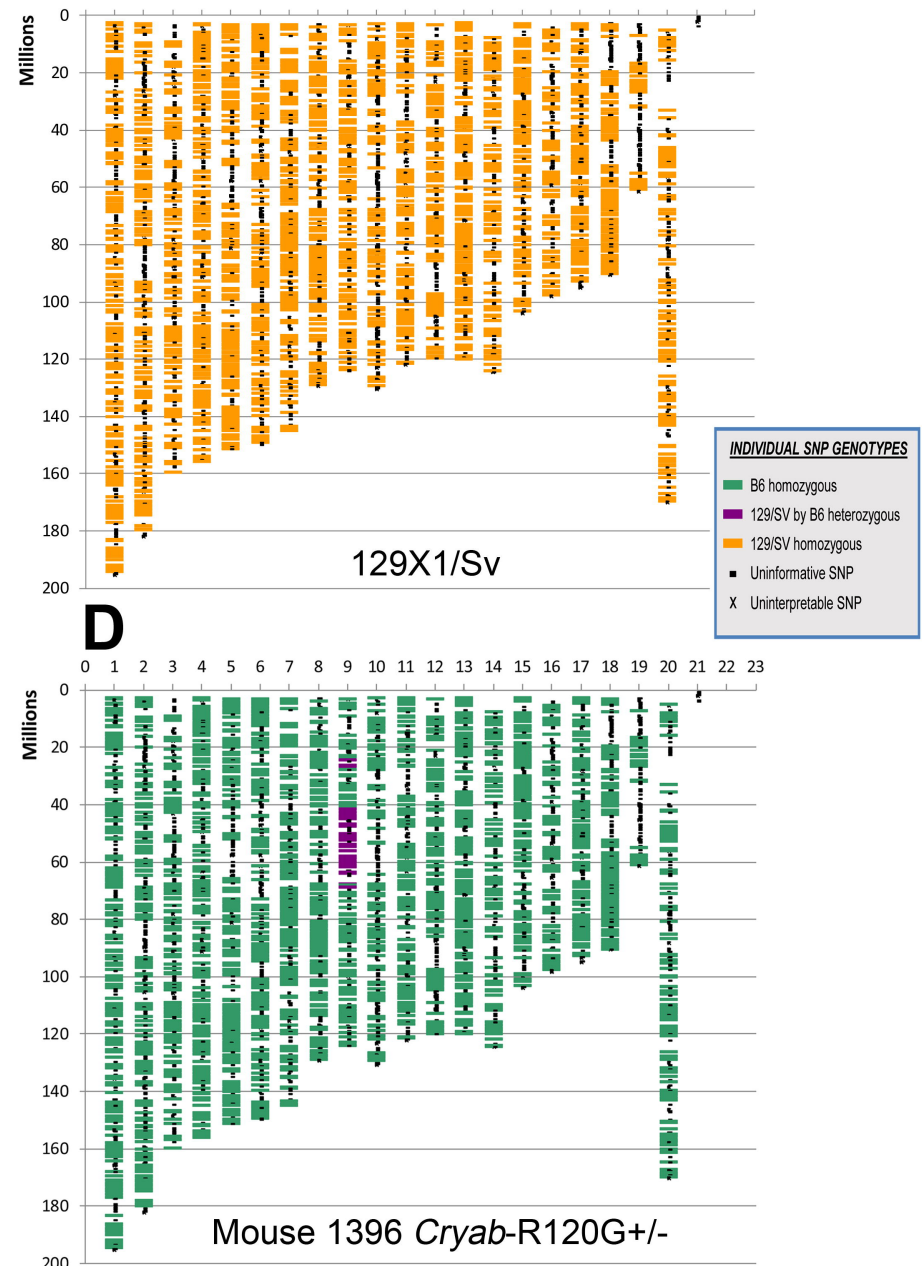
